\title{
In vivo kinematics of medial unicompartmental osteoarthritic knees during activities of daily living
}

\author{
Francesco Fiacchi, Francesco Zambianchi*, Vitantonio Digennaro, Ippazio Ricchiuto, Raffaele Mugnai, \\ Fabio Catani
}

Department of Orthopaedic Surgery, Azienda Ospedaliero-Universitaria Policlinico di Modena, University of Modena and Reggio-Emilia, Modena, Italy

\section{A R T I C L E I N F O}

\section{Article history:}

Received 2 June 2014

Received in revised form 13 August 2014

Accepted 18 August 2014

\section{Keywords:}

Kinematics

Unicompartmental

Osteoarthritis

Knee

Video-fluoroscopy

\begin{abstract}
A B S T R A C T
Few studies exist describing unicompartmental osteoarthritic knee kinematics. Moreover, the role of the anterior cruciate ligament $(A C L)$ in the determination of knee kinematics has not been fully described. The objective of the current study was to analyze the in vivo kinematics of knees with medial osteoarthritis (OA) and intact ACL during closed and open chained motion.

Eight patients scheduled for UKA diagnosed with primary medial OA underwent knee CT-scans and video-fluoroscopy. Fluoroscopic analysis included stair climbing, chair rising and leg extension. Threedimensional bone positions were obtained from each image by iterative procedures using a CADmodel-based shape-matching technique. Patterns of axial rotation and anterior-posterior (AP) motion of the medial and lateral femoral condyle were obtained with specific software.

The femur reported an overall external rotation relative to the tibia from extension to flexion in all tasks. Average AP translation of the medial femoral condyle were smaller in open-chained tasks than in weight-bearing conditions. Average AP motion of the lateral femoral condyle reported an overall posterior translation with knee flexion.

The absent natural "screw-home" mechanism and the lack of medial condyle posterior translation was explained by bone-cartilage defects and meniscal degeneration. Relevant findings were the kinematic pattern differences between weight-bearing and open chained activities, suggesting that in biphasic muscle contraction and unloaded conditions, the function of the cruciate ligaments was not physiological. The kinematics of knees with medial OA and intact ACL differed from healthy knees.
\end{abstract}

(c) 2014 Elsevier B.V. All rights reserved.

\section{Introduction}

The evaluation of in vivo knee kinematics is important in order to understand the effects of joint injury, diseases and in order to improve the outcome of surgical procedures.

Video-fluoroscopic analysis has been used for 20 years to determine knee motion from fluoroscopic image sequences [1,2], both for patients operated with total knee arthroplasty (TKA) $[3,4]$ and more recently for motion measurements in normal or osteoarthritic joints.

Healthy knee kinematics has been assessed with videofluoroscopic analyses to understand the deviations of the kinematics after surgical treatment [5]. Moro-oka et al. described healthy knee kinematics during the activities of squatting, stair climbing and kneeling and reported an overall femoral external rotation relative to the tibia with flexion. Anterior-posterior (AP) translation of the condylar contact regions showed three phases over the range of motion according to the performed activity: sharp femoral external rotation from $0^{\circ}$ to $20^{\circ}$ flexion (squatting) - the so-called "screw-home" mechanism - with posterior translation

\footnotetext{
* Corresponding author at: Department of Orthopaedic Surgery, Azienda Ospedaliero-Universitaria Policlinico di Modena, University of Modena and Reggio-Emilia, via del Pozzo 71, 41124 Modena, Italy. Tel.: +39 059422 4309; fax: +39059 4224313 .

E-mail address: francesco.zambianchi@gmail.com (F. Zambianchi).
}

of the medial and lateral contact points (CP). Relatively constant rotation and posterior translation of both condyles from $20^{\circ}$ to $80^{\circ}$ (stair climbing) and an increasing femoral external rotation from $80^{\circ}$ to $150^{\circ}$ flexion (kneeling) resulted predominantly from posterior lateral translation, essentially pivoting about the medial compartment. In this range of flexion, medial AP tibio-femoral translation was reduced, determining a medial pivot point kinematics [5].

Few studies have highlighted the kinematics of knees with unicompartmental osteoarthritis. Knees with medial osteoarthritis (OA) have shown somewhat similar kinematic patterns to those of the normal knee, such as femoral external rotation gradually increasing with flexion for squatting and kneeling, and gradually decreasing with extension for stair climbing [6]. Nevertheless, some differences have been described between medial osteoarthritic and healthy knees. The osteoarthritic joint starts knee flexion at reduced values of femoral external rotation, which is maintained during all the range of motion. Moreover, in knees with medial OA the natural screw-home movement has not been described and medial and lateral femoral condyle CP on the tibial plateau have not exhibited significant posterior translation, especially between $30^{\circ}$ and $80^{\circ}$ of knee flexion [6].

In the literature, the role of the anterior cruciate ligament $(\mathrm{ACL})$ and its presence or absence in the determination of knee kinematics has not been clarified yet. For this reason, it's the authors' belief that function of the ACL, the articular surface 
geometry and the menisci should be investigated to better understand the overall knee kinematics.

The objective of the present study was to analyze the in vivo kinematics of knees with medial OA with intact ACL during closed and open chain motions. The kinematics of pathologic knees may influence the kinematics after unicompartmental knee arthroplasty (UKA). It is the author's belief that clarification of the differences in the kinematics between $\mathrm{OA}$ and normal knees is very important for enhancing implant design and positioning. It was hypothesized that the kinematic of knees with medial OA differs from healthy knees, showing similar patterns of axial rotation and reduced posterior translation of the femoral condyles, due to cartilage wear and menisci degeneration.

\section{Material and methods}

Eight consecutive patients scheduled for robotic UKA at the orthopaedic department of Modena University Hospital, Modena, Italy, between January 2013 and May 2014 were prospectively selected. All patients gave written informed consent prior to being included in the study, which was performed in accordance with the Ethical Standards of the 1964 Declaration of Helsinki, as revised in 2000. Patients with diagnosis of rheumatoid arthritis or post-traumatic knee arthritis were not included in the cohort. The mean age of the selected cohort was 70.4 (SD 7.9; range 60-85) years; four patients were males, four were females.

Primary medial unicompartmental knee osteoarthritis (Ahlbäck stage III-IV) was the diagnosis in all cases. Pre-operative clinical examination showed ACL integrity in all patients. This inclusion criterion was verified and confirmed intra-operatively during surgery. In all knees, cartilage destruction in the medial compartment on the femur and tibia was severe, with subchondral bone exposure and medial meniscus degeneration.

All patients underwent knee CT scan and video-fluoroscopy examination as standard pre-operative protocol. A General Electric 64 multislice CT with a collimation of 0.75 was used in all patients; knee CT scans were performed perpendicular to the long axis of the lower limb, with a slice thickness of $0.63 \mathrm{~mm}$. From CT scan images, three-dimensional CAD models of the ostheoarthritic femur and tibia were eventually created using two open source softwares (ITK-Snap, Penn Image Computing and Science Laboratory, University of Pennsylvania, PN, USA and MeshLab, Computer Science Department of Pisa University, Pisa, Italy). Anatomic coordinate systems were embedded in each bone following conventions [5,7].

Fluoroscopy analysis was performed on the same day of CT scan acquisition during three activities of daily living: stair climbing, chair rising and leg extension. For stair climbing, three $21-\mathrm{cm}-$ high steps were used; to assess stepping up, only the first step was used, and three up/down cycles were performed in a single repetition and the ascent phase was captured. For the rising, chair height was set specifically for each patient in order for him or her to begin with the knee flexed at about $80^{\circ}$. Even for this task, only the ascent phase was analyzed. For the extension against gravity task, patients were examined in a semi-supine position (i.e. sitting at $45^{\circ}$ ), with their knee hanging as flexed as possible over the end of the examination chair. Data collection and analysis procedures were as previously reported [8-10] and included the use of a standard flat panel detector (Opera Swing, GMM S.p.a, Italy) with 15 images per second. Three-dimensional bone positions and orientations were obtained from each fluoroscopic image by an iterative procedure using a CAD-model-based shapematching technique [1] (Fig. 1). Condylar contact was assumed on the medial and lateral compartments as the two sets of points at minimum distance between the femoral condyles and the tibial plateau. The positions of these $\mathrm{CP}$ were expressed in the tibial

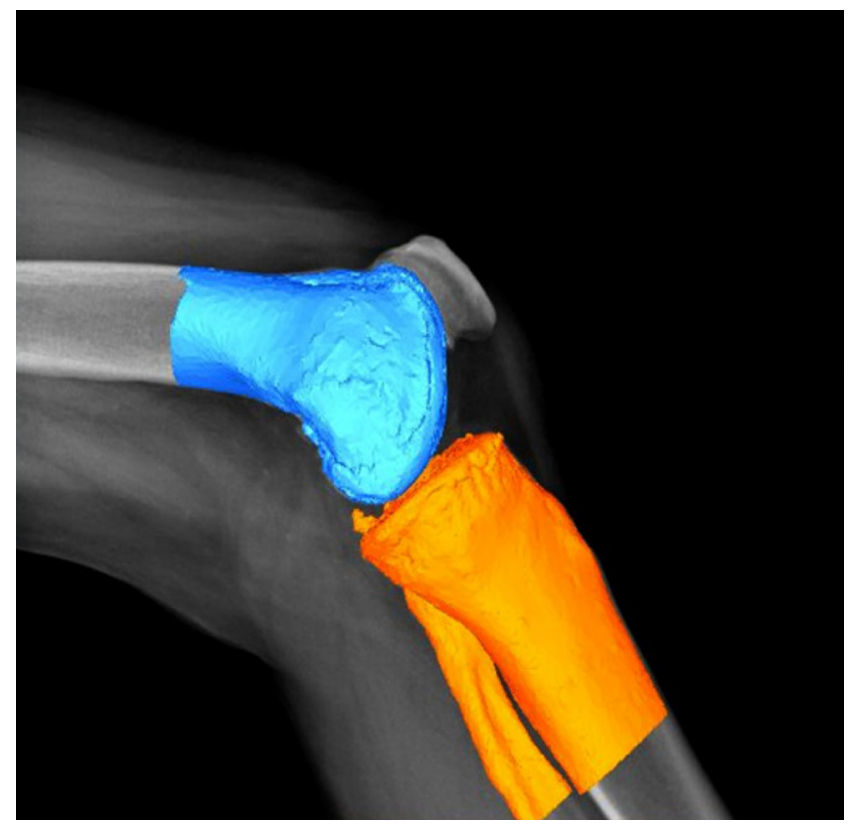

Fig. 1. 3D-to-2D CT model-to-flat panel image registration was used to determine the kinematics of medial OA knees during three activities of daily living.

plateau reference frame in terms of locations over the anteriorposterior length. Patterns of AP motion of the $\mathrm{CP}$ were therefore obtained independently for the medial and lateral condyles. Contact-line rotation, defined as rotation of the line connecting the medial and lateral $\mathrm{CP}$ with respect to the mediolateral axis on the tibial transverse plane, was calculated for each flexion angle, but here, it is reported over predefined flexion angles with $10^{\circ}$ increments. Continuous data are here expressed as means and standard deviations (SD); categorical data are presented as absolute numbers.

\section{Results}

The femur reported an overall external rotation relative to the tibia from extension to flexion in all three motor tasks (Fig. 2). Based on the bone-embedded coordinate systems, femoral external rotation at minimal and maximal flexion (from $5^{\circ}$ to $75^{\circ}$ of knee flexion) resulted on average $14^{\circ}$ (SD $4^{\circ}$ ) during chair rising (range $-4^{\circ}$ to $\left.+10^{\circ}\right), 9^{\circ}\left(\mathrm{SD} 3^{\circ}\right)$ during the stair climbing task (range $-3^{\circ}$ to $\left.+6^{\circ}\right)$, and $6^{\circ}\left(\operatorname{SD~} 1^{\circ}\right.$ ) during leg extension (range $-3^{\circ}$ to $+3^{\circ}$ ). Similar absolute values of femoral axial rotation were observed in chair rising and stair climbing from $10^{\circ}$ to $70^{\circ}$ of knee flexion.

Average AP translations of the medial femoral condyle CP were smaller in leg extension than in chair rising and stair climbing (Fig. 3). For chair rising, the average translation of the medial femoral condyle on the tibial plateau was $11 \mathrm{~mm}$ (SD 4; range 4-15 mm). For stair climbing, the average AP translation was 5 $\mathrm{mm}$ (SD 2; range 5-10 $\mathrm{mm}$ ); in leg extension, the reported average AP translation was $5 \mathrm{~mm}$ (SD 2; range 2-7 $\mathrm{mm}$ ).

Average AP translations of the lateral femoral condyle $\mathrm{CP}$ were smaller in leg extension than in chair rising and stair climbing (Fig. 4). For chair rising, the average translation of the lateral femoral condyle on the tibial plateau was $15 \mathrm{~mm}$ (SD 5; range -7 to $8 \mathrm{~mm}$ ). For stair climbing, the average AP translation was 14 $\mathrm{mm}$ (SD 4; range -7 to $7 \mathrm{~mm}$ ); in leg extension, the average AP translation was $4 \mathrm{~mm}$ (SD 1 ; range $1-4 \mathrm{~mm}$ ).

In the stair climbing and chair rising tasks, the medial condyle $\mathrm{CP}$ showed a relatively small posterior translation from $10^{\circ}$ to $50^{\circ}$ of flexion, with a greater anterior translation from $50^{\circ}$ to $75^{\circ}$. In leg extension, the medial femoral condyle $\mathrm{CP}$ showed a 


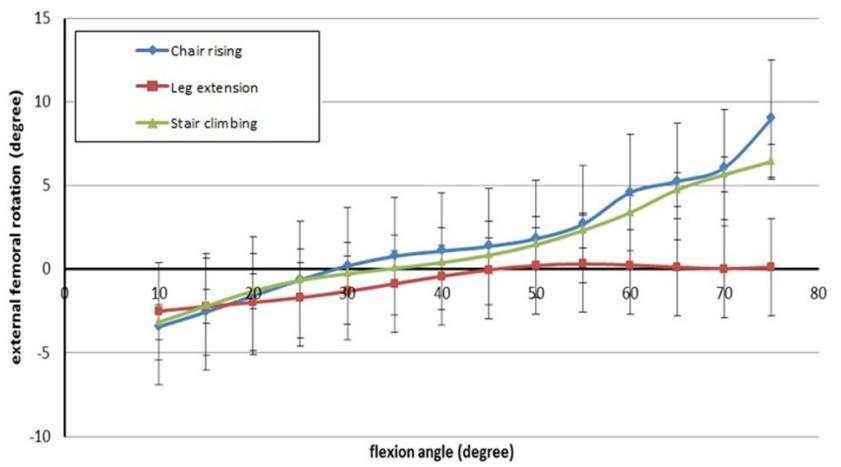

Fig. 2. Femoral axial rotation in the three motor tasks at different knee flexion degrees.

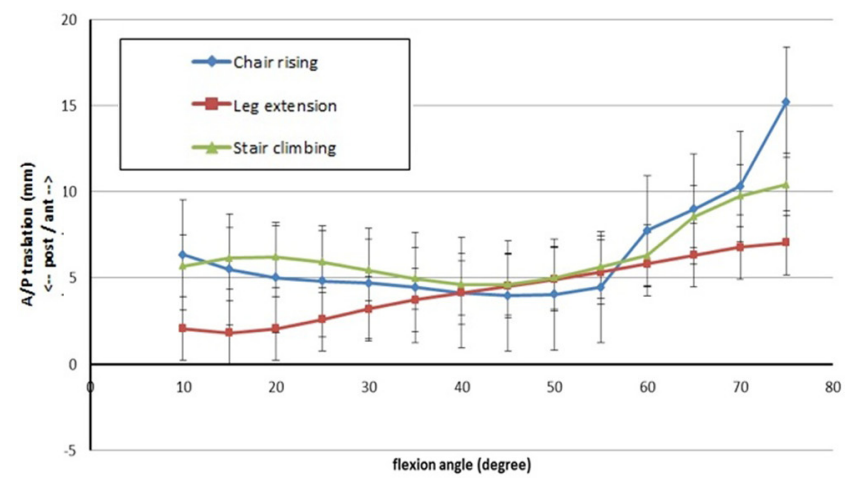

Fig. 3. AP translation of the medial femoral condyle on the tibial plateau in the three motor tasks at different knee flexion degrees. Ascending curves are representative of femoral condyle anterior translation on the tibial plateau.

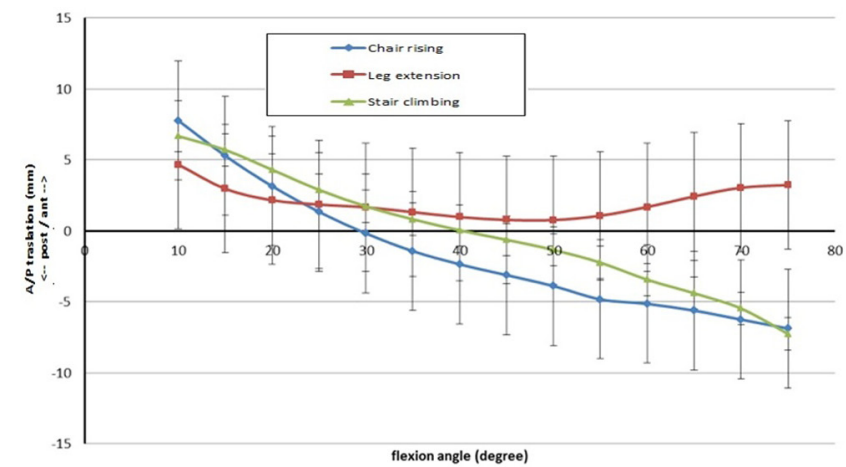

Fig. 4. AP translation of the lateral femoral condyle on the tibial plateau in the three motor tasks at different knee flexion degrees. Ascending curves are representative of femoral condyle anterior translation on the tibial plateau.

progressive anterior translation from $10^{\circ}$ to $75^{\circ}$. In stair climbing and chair rising, the lateral femoral condyle $\mathrm{CP}$ showed a progressive posterior translation from $10^{\circ}$ to $75^{\circ}$ of knee flexion. In leg extension, the lateral condyle $\mathrm{CP}$ showed a relatively small posterior translation from $10^{\circ}$ to $50^{\circ}$, with a small anterior translation from $50^{\circ}$ to $75^{\circ}$.

In all three motor tasks, a medial pivot point was observed (Fig. 5).

\section{Discussion}

The most important finding of the present study is the demonstration that the kinematic pattern of knees with medial unicompartmental $\mathrm{OA}$ and intact $\mathrm{ACL}$ differs from the kinetic

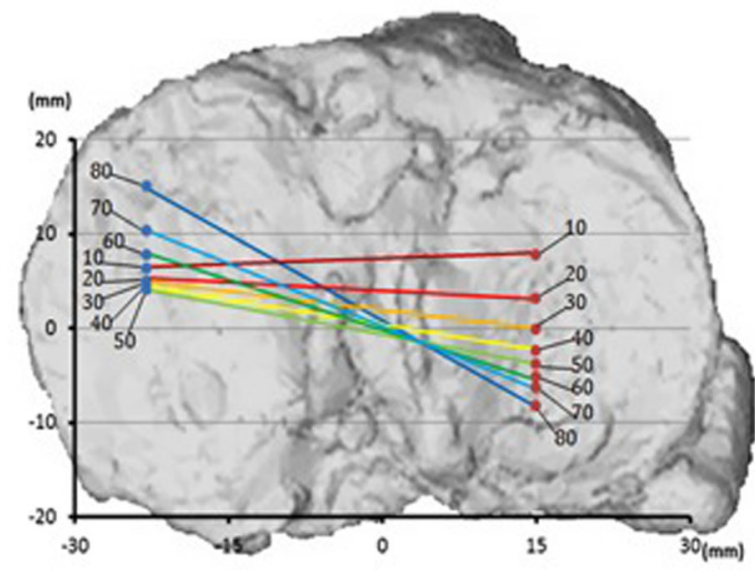

\section{Chair rising/sitting contact points}

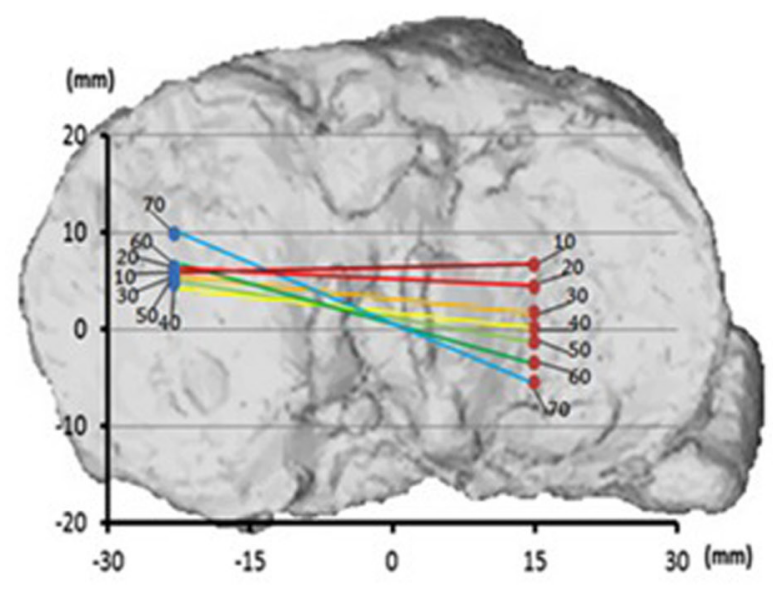

Stair climbing/descending contact points

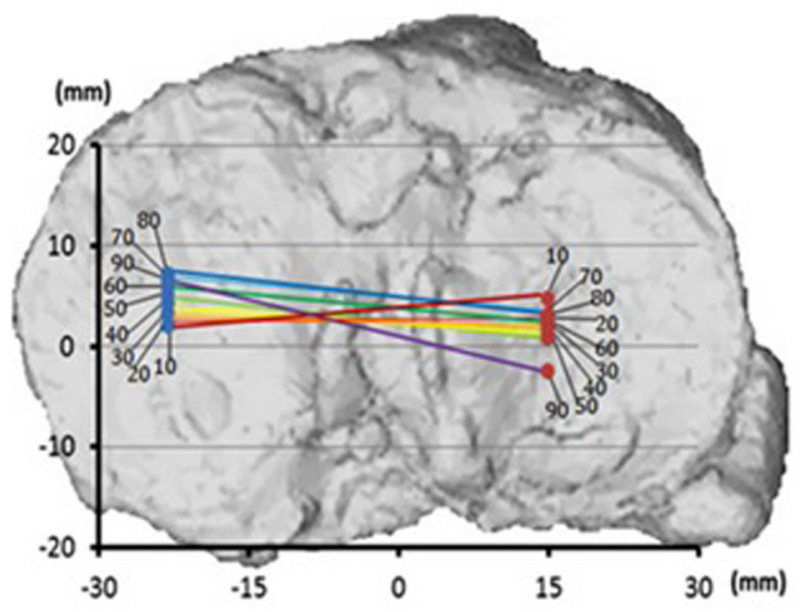

Leg extension/flexion contact points

Fig. 5. Average contact locations overimposed on a right tibial surface model of a representative knee with medial OA. Average contact locations are shown for $10^{\circ}$ flexion increments for each motor task.

trend of the healthy knee [5]. In the present work, the dynamic 3D kinematic of OA knees was examined during three activities, including weight-bearing and open chained motor tasks. 
Different values of knee axial rotation were observed in the three activities, with the greatest external rotation of the femur relative to the tibia reported during weight-bearing activities (stair climbing and chair rising) and reduced average values of femoral external rotation during open chained motor task (leg extension). The data describing the stair-climbing task were eventually compared with those by Moro-oka et al. [5] in healthy joints during the same motor task and analyzed with the same methodology. From $10^{\circ}$ to $75^{\circ}$ of knee flexion, an overall $9^{\circ}$ femoral external rotation was reported both in healthy and OA knees (Fig. 2) [5]. However, at every degree of knee flexion, the OA knee axial rotation curve displayed a deviation of about $10^{\circ}$ compared to the axial rotation of the healthy joint. This finding has already been noted by Matsui et al., who evaluated the femoro-tibial rotation using CT and reported that the femur tended to locate in a relative internal rotation position in knees with severe OA compared to normal joints [11], and by Hamai et al. in their video-fluoroscopic analysis of the kinematics of the osteoarthritic knee [6]. Moreover, in the present series the natural "screw-home" mechanism which occurs between $0^{\circ}$ to $20^{\circ}$ of healthy knee flexion - was not observed, presumably because the patients could not reach full extension. This functional limitation is attributable to cartilage degeneration, osteophytes and menisci disease in the medial compartment of unicompartmental arthritic knees. The reduction of the sharp internal rotation of the femur relative to the tibia in the last degrees of extension in the osteoarthritic joint has already been described by Nagao et al., who investigated normal and OA knees with ultrasound and found that tibial external rotation at maximal extension and femoral rotation of the "screw-home" movement proportionately decreased with progression of medial compartment osteoarthritis [12].

The AP motion of the osteoarthritic knee has not been clearly described in the literature so far. The presented results showed that the medial femoral condyle $\mathrm{CP}$ described from $10^{\circ}$ to approximately $50^{\circ}$ of knee flexion reduced motion on the tibial plateau and an overall anterior translation from mid to full flexion in weight-bearing motor tasks. On the other hand, in open chained motor task the medial condyle reported a progressive anterior translation throughout the range of motion (Fig. 3). As described by Hamai at al., the lack of a posterior translation of the medial condyle may be caused by soft tissue contractures and osteophytes formation with cartilage-bone erosion in the medial compartment and may be related to the cartilage wear pattern on the tibial plateau $[6,13]$. Moreover, the anterior translation of the medial femoral condyle with knee flexion - and its backward movement with extension - can be assumed as responsible for cartilage and meniscus damage due to increased shear forces on the articular surfaces. In accordance with Moschella et al., the overall of the femoral condyle $\mathrm{CP}$ translation on the medial tibial plateau seems to be strictly dependent on ACL integrity. As reported by Moschella et al. in their study of tibial plateau wear patterns, cartilage erosion on OA knees with intact ACL occurred in the central to medial region of the medial plateau, despite of OA knees with ruptured ACL, which showed larger posterior wear [13] and tended to display more posterior contact during weight bearing activities as reported by Hamai et al. in their videofluoroscopy analysis [6].

Regarding the translation of the lateral CP, an overall posterior motion of the femoral condyle was displayed. This contributes to create a medial pivot type of axial rotation pattern (Fig. 5), in which the femur externally rotates relative to the tibia as flexion progresses, as explained above. The AP translation of the lateral femoral condyle noted a progressive and remarkable posterior motion for weight-bearing activities and a small relative motion during leg extension/flexion task (Fig. 4). The described results are comparable to those presented by Moro-oka et al. and Lu in the healthy knee $[5,14]$. The relative motion of the lateral condyle on the tibial plateau seems to be independent from osteoarthritic degeneration. Not even the ACL integrity seems to be a factor with major influence on the translation of the lateral femoral condyle. In fact, as described by Dennis et al., who compared healthy with ACL deficient knees, subjects with or without functional cruciate ligament reported similar posterior motion patterns [15].

Relevant findings of the present study were the kinematic pattern differences displayed between weight-bearing activities (chair rising and stair climbing) and open chained motor tasks, with smaller magnitude values for femoral axial rotation and medial and lateral AP translation reported by the leg extension motion. These findings are consistent with those of Lu et al. who compared the motion pattern of eight knees in conditions of load and unload [14]. The presented data display that in biphasic muscle contraction and unload conditions the function of the anterior cruciate ligament is not physiological. Nevertheless, it is yet to be acknowledged the influence of a loosened ACL and the degenerative changes in the bearing surfaces on the joint motion. However, the presented results appear to suggest different effects of load on the overall knee kinematics.

Several limitations of the present study need to be discussed. First, the current approach to analyzing joint contact ignores the degenerate menisci, which are invisible on radiographs but obviously affect joint contacts and load distribution. Moreover, the lowest CP are created starting from CT-derived models, which assume a uniform cartilage thickness. For osteoarthritic knees, models registration should be performed using CT-derived images for bone and MRI-derived pictures for cartilage surfaces. This approach would impose a bigger burden on preoperative subjects. Third, single-plane imaging has more uncertainties on the mediolateral translations. Lastly, the current study included only eight cases and therefore can't be considered completely representative of knees with unicompartmental medial OA. Further study should be performed on a larger cohort of patients to confirm the presented findings.

\section{Conclusion}

Kinematics in knees with unicompartmental OA and intact ACL differs from healthy knee motion patterns. Pathological changes, such as cartilage wear, menisci degeneration and osteophytes influence knee kinematics. Load and unload conditions seem to have a role in the determination of the overall joint kinematics. These findings can be useful for enhancing UKA design.

\section{Conflict of interest statement}

All the authors certify that they do not have any conflict of interest relating to this paper.

\section{References}

[1] Banks SA, Hodge WA. Accurate measurement of threedimensional knee replacement kinematics using single-plane fluoroscopy. IEEE Trans Biomed Eng 1996;43:638-49.

[2] Walker SA, Hoff W, Komistek R, Dennis D. "In vivo" pose estimation of artificial knee implants using computer vision. Biomed Sci Instrum 1996;32:143-50.

[3] Dennis DA, Komistek RD, Mahfouz MR, Haas BD, Stiehl JB. Multicenter determination of in vivo kinematics after total knee arthroplasty. Clin Orthop 2003;416:37-57.

[4] Victor J, Banks S, Bellemans J. Kinematics of posterior cruciate ligamentretaining and -substituting total knee arthroplasty: a prospective randomised outcome study. J Bone Joint Surg [Br] 2005;87:646-55.

[5] Moro-oka TA, Hamai S, Miura H, Shimoto T, Higaki H, Fregly BJ, et al. Dynamic activity dependence of in vivo normal knee kinematics. J Orthop Res 2008;26:428-34.

[6] Hamai S, Moro-oka T, Miura H, Shimoto T, Higaki H, Fregly BJ, et al. Knee kinematics in medial osteoarthritis during in vivo weight-bearing activities. J Orthop Res 2009;27:1555-61. 
[7] Moro-oka TA, Hamai S, Miura H, Shimoto T, Higaki H, Fregly BJ, et al. Can MR derived bone models be used for accurate motion measurement with singleplane 3D shape registration? J Orthop Res 2007; 25:867-72.

[8] Catani F, Ensini A, Belvedere C, Feliciangeli A, Benedetti MG, Leardini A, Giannini S. In vivo kinematics and kinetics of a bi-cruciate substituting total knee arthroplasty: a combined fluoroscopic and gait analysis study. J Orthop Res 2009; 27(12):1569-75.

[9] Catani F, Belvedere C, Ensini A, Feliciangeli A, Giannini S, Leardini A. In-vivo knee kinematics in rotationally unconstrained total knee arthroplasty. J Orthop Res 2011; 29(10):1484-90.

[10] Fantozzi S, Catani F, Ensini A, Leardini A, Giannini S. Femoral rollback of cruciate-retaining and posterior-stabilized total knee replacements: in vivo fluoroscopic analysis during activities of daily living. J Orthop Res 2006;24:2222-9.
[11] Matsui Y, Kadoya Y, Uehara K, Kobayashi A, Takaoka K. Rotational deformity in varus osteoarthritis of the knee: analysis with computed tomography. Clin Orthop 2005;433:147-51.

[12] Nagao N, Tachibana T, Mizuno K. The rotational angle in osteoarthritic knees. Int Orthop 1998;22:282-7.

[13] Moschella D, Blasi A, Leardini A, Ensini A, Catani F. Wear patterns on tibial plateau from varus osteoarthritic knees. Clin Biomech 2006;21:152-8.

[14] Lu T, Tsai T, Kuo M, Hsu HC, Chen HL. In vivo three-dimensional kinematics of the normal knee during active extension under unloaded and loaded conditions using single-plane fluoroscopy. Med En Phys 2008;30:1004-12.

[15] Dennis D, Mahfouz M, Komistek R, Hoff W. In vivo determination of normal and anterior cruciate ligament-deficient knee kinematics J Biomech 2005;38:241-53. 